\title{
Quelle place pour l'imagination dans une phénoménologie de l'architecture ? Réflexions à partir de Roman Ingarden
}

\author{
Par RUDY STEINMETZ \\ Université de Liège
}

Résumé En raison des contraintes matérielles et fonctionnelles qui pèsent sur l'architecture, Roman Ingarden a tendance à n'accorder qu'une place restreinte à l'imagination dans ce domaine artistique. Or de grands architectes modernes comme Le Corbusier et Mies van der Rohe ont tâché de libérer l'espace construit de telles contraintes. Pour y parvenir, ils ont mis en œuvre ce qu'il n'est pas inconvenant d'appeler une épokhè architecturale. L'effet le plus notable de celle-ci a consisté dans la « réduction » de la substance physique et des finalités utilitaires des structures bâties qu'ils portaient à l'existence. C'est cette épokhè et la valorisation du rôle de l'imagination qu'elle entraîne dans le champ architectural qui sont au cœur de l'analyse proposée ici.

\section{Ingarden et l'évincement de l'imagination en architecture}

Dans L'œuvre architecturale, ouvrage assez peu fréquenté paru en 1945, mais qui fut mis en chantier dès l'année 1928, Roman Ingarden poursuit trois objectifs, comme Patricia Limido-Heulot l'a rappelé dans la présentation de sa traduction française ${ }^{1}$. Il s'agit, pour lui, tout d'abord, de vérifier si la structure feuilletée ou stratifiée de l'œuvre d'art, telle que son analyse de la littérature l'avait initialement dégagée, s'applique ou non au cas de l'architecture. Il s'agit, ensuite, à la faveur de cet examen, de mettre en évidence la

${ }^{1}$ Cf. P. Limido-Heulot, Introduction à Roman Ingarden, L'œuvre architecturale, 1945, Paris, Vrin, 2013, p. 7-35. 
spécificité de l'œuvre édifiée dont il fut, avec Heidegger, l'un des premiers à jeter les bases d'une approche phénoménologique. Il s'agit, enfin, de relancer, une nouvelle fois, sur le terrain de l'art de bâtir, la querelle entre le réalisme dont il se veut le défenseur et l'idéalisme de Husserl qu'il n'a eu de cesse de remettre en cause, en particulier au cours de ses investigations dans le domaine de l'esthétique.

Ce qui est frappant et qui ne doit pas manquer d'attirer l'attention, c'est que, dans la poursuite de ce triple objectif, auquel on s'attachera plus tard, Ingarden se montre très discret - pour ne pas dire muet — quant à la place et à la fonction de l'imagination, tant en ce qui concerne la création de l'œuvre architecturale qu'en ce qui concerne sa réception. Le motif de cette discrétion nous paraît devoir être mis en relation avec le privilège qu'Ingarden accorde à la dimension matérielle de l'objet construit, à sa réalité chosale. On ne saurait lui donner tort : un bâtiment est avant tout une entité physique qui s'impose à nous par l'immédiateté de sa présence. Il fait partie intégrante de notre environnement. Il le module et module notre vie. Si, pareil en cela à celui de la sculpture, l'espace architectural est un espace à trois dimensions, il a toutefois ceci de particulier qu'il est un espace enveloppant, englobant, un espace qui intègre en lui notre propre existence. L'architecture étend sur nous les relations spatiales qu'elle institue en raison du fait qu'elle «n'est pas fermée sur soi ni isolée du monde réel $»^{1}$, mentionne Ingarden. Je ne peux jamais être exclusivement en position de spectateur désintéressé dans mon rapport à l'édifice bâti. Même quand je suis devant lui, je suis inclus dans le rayonnement de sa spatialité. Mais, je suis aussi en lui, en contact tactile, et même multi-sensoriel, avec lui : je touche son sol ; je respire ses odeurs ; je vibre à ses sonorités ; je détecte ses variations lumineuses: «L'édifice me parle d'une manière plus physique que les autres arts ", écrivait Henri van Lier, en 1959, dans Les arts de l'espace ${ }^{2}$. Ingarden ne l'aurait certainement pas démenti.

Que l'on y prenne garde : nous ne sommes pas en train de prétendre que, pour Ingarden, l'œuvre architecturale se résume à son effectivité, qu'une construction est un être réel et seulement un être réel. Mais, plutôt, que, selon le parti-pris qu'il adopte, l'architecture est prioritairement un être réel, quoique, une fois encore, elle ne se réduise pas à sa réalité matérielle. On y reviendra par la suite.

Deux conséquences découlent de cette primauté reconnue par Ingarden à l'objectalité de la chose architecturale. Premièrement: l'architecture est,

\footnotetext{
${ }^{1}$ R. Ingarden, op. cit, p. 101.

${ }^{2}$ H. van Lier, Les arts de l'espace, 1959, Tournai, Casterman, 1971, p. 232.
} 
par excellence, le domaine artistique où le réalisme duquel il se revendique se manifeste de la manière la plus nette et où l'écart vis-à-vis de l'idéalisme husserlien se creuse irrémédiablement. Car, nul ne l'ignore, Husserl fait de l'objet d'art un objet idéal, un objet irréel qui mobilise l'imagination, laquelle met hors-jeu toute espèce de considération portant aussi bien sur l'effectivité que sur le caractère pratique d'un tel objet ${ }^{1}$. Et cela même quand il s'agit d'objets d'usage ${ }^{2}$. Seule leur apparence entre en ligne de compte. Point crucial sur lequel Ingarden ne manque pas de s'éloigner de la position de son maître dans la conception qui est la sienne de l'ouvrage d'architecture. Deuxièmement : confrontée à la solidité, à la consistance de la chose édifiée qui est là, massive, pleinement offerte dans sa robuste concrétude, l'imagination se trouve, chez Ingarden, comme « médusée », "pétrifiée », destituée de son pouvoir. Un pouvoir que Husserl définit par l'aptitude à jouer avec les apparences sensibles, à explorer ou à exploiter les latences, les possibles que renferme notre expérience du réel, laquelle étant partielle, fragmentaire, se trouve fondée sur un horizon ouvert d'esquisses potentielles, d'aspects implicites que l'imagination peut alors se donner pour tâche, précisément, de figurer ou de préfigurer sur le mode de la «quasi-positionnalité » ou du « comme si »" ${ }^{3}$. Cette capacité que Husserl reconnaît à la Phantasia de traiter avec les lacunes, les failles du réel, de pallier à ses déficiences, d'en dégager les intentions inexposées, Ingarden tend, au contraire, à la lui soustraire dans ses considérations sur l'art d'édifier. La discrétion dont il enveloppe l'imagination étant, de sa part, symptomatique de cette soustraction, de cette minorisation du rôle qu'il refuse de lui attribuer.

On trouvera des preuves de ce que nous avançons dans les quelques passages significatifs de L'œuvre architecturale où intervient le problème de la création de l'espace construit (le même constat pouvant être tiré à propos de son appréhension ainsi qu'on le découvrira plus loin). Tous ces passages sont frappés au sceau de la même idée : la forme extérieure que prend un bâtiment, loin de pouvoir être laissée à la libre élaboration imaginative de son ou de ses auteurs, doit être en accord avec l'organisation structurelle qui en

\footnotetext{
${ }^{1}$ Cf. E. Husserl, «Phantasia et conscience d'image », 1904/1905, dans Phantasia, conscience d'image, souvenir. De la phénoménologie des présentifications intuitives. Textes posthumes (1898-1925), Grenoble, Millon, 2002, p. 168.

${ }^{2}$ Ibid., p. 168, note 253. L'influence de Kant est ici déterminante. Husserl le reconnaît lui-même.

${ }^{3}$ E. Husserl, Méditations cartésiennes, 1950, Paris, PUF, 1994, § 25, p. 104. Voir, sur cet aspect, Annabelle Dufourcq, La dimension imaginaire du réel dans la philosophie de Husserl, Dordrecht, Springer, 2011, ch. 4, p. 113-124 ; ch. 5, p. 125134.
} 
rend l'usage possible. Qu'un tel accord vienne à manquer, qu'une « disharmonie avec le but du bâtiment ${ }^{1}$, déplore Ingarden, frappe la configuration formelle de ce dernier et nous n'avons plus affaire, poursuit-il, qu'à « une œuvre ratée, un monstre $»^{2}$, en ceci que, précise-t-il, «l'œuvre est alors privée d'unité intime et ne peut conduire à la constitution d'aucun objet esthétique unitaire $»^{3}$.

Il apparait clairement, à la lueur de la citation précédente, qu'il y a, chez Ingarden, une « logique interne ${ }^{4}$ qui préside à la réalisation de l'œuvre architecturale. Cette logique rogne les ailes de l'imagination de l'architecte. Elle l'oblige à tenir compte de la nécessité du fait que l'architecture en tant qu'art consiste, avant tout, dans la production d'un objet réel soumis à des impératifs d'ordre aussi bien technique qu'utilitaire, impératifs avec lesquels toute visée esthétique doit s'accorder sous peine d'échec. Si l'on en croit Ingarden, pour reprendre la devise célèbre de Louis Henri Sullivan, figure de proue de l'école de Chicago, «la forme suit la fonction » («Form follows function »). Façon de dire qu'elle s'y asservit.

Une nouvelle mise en garde s'avère ici indispensable: de la même façon que l'architecture, on l'a dit, n'est pas qu'une entité déterminée matériellement, il faut ajouter qu'elle n'est pas non plus, dans l'esprit d'Ingarden, qu'une entité déterminée fonctionnellement. Ce qui est primordial en elle, selon l'angle de vue qu'il adopte, c'est qu'elle est essentiellement une chose concrète et utile, avant d'être et même pour être un objet d'appréciation esthétique. Nous nous pencherons sur ce problème plus loin.

On trouve de cette prévalence physico-technique un indice supplémentaire dans ce que Ingarden déclare, toujours à propos de la conception architecturale. Celle-ci, remarque-t-il, est, de façon intrinsèque, soustraite à la contrainte représentative. L'image (maquette, dessin, etc.) produite par l'architecte n'est pas la réduplication d'un modèle préexistant ; elle ne figure pas son référent, elle l'engendre. Et, par là même, elle renverse la logique iconique en ce qu'elle fait exister la représentation imagée avant le réel dans la dépendance duquel nous avons habituellement tendance à la situer (l'image est image de quelque chose). "La forme spatiale de l'œuvre architecturale (...) n'est en rien un figuré, mais plutôt quelque chose qui se

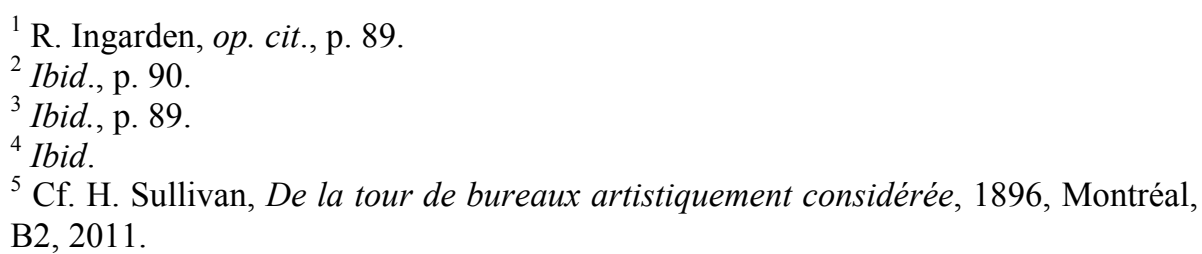


présente en personne (...) à l'observateur. ${ }^{1}{ }^{1}$ Elle est, avec la musique et les arts abstraits ${ }^{2}$, un «jeu de pure auto-manifestation $»^{3}$, d'exhibition de ses propres qualités sensibles. Et pourtant, malgré cette marge de manœuvre créatrice apparemment illimitée que lui reconnaît Ingarden, il convient, assène-t-il, qu'elle sache respecter les «lois des lourdes masses » ${ }^{4}$, c'est-àdire les lois physiques qui, en plus de son utilité, conditionnent sa solidité et qu'il lui revient d'exprimer formellement. La vérité de l'architecture est donc bien à rechercher, quant à la conviction d'Ingarden, prioritairement, dans sa physicalité et sa fonctionnalité, et non dans la liberté créatrice de formes pouvant s'émanciper de cette double contrainte.

On le voit bien, les lois physiques et structurelles marquent de leur empreinte la production architecturale telle qu'Ingarden l'envisage. Par opposition à la liberté imaginative du poète qui n'a pas de frein, les astreintes techniques et utilitaires pesant sur l'architecte orientent son art vers la prise en compte de son ancrage dans la factualité de l'existence :

Tout le domaine, prétend Ingarden, de ce qui est simplement impossible à l'architecte est ouvert à l'imagination poétique. Mais, nuance-t-il aussitôt, la fondation de l'œuvre architecturale dans une chose matérielle lui donne en revanche une plénitude et une concrétude qu'aucune œuvre littéraire pure ne peut atteindre 5 .

C'est en vertu de cette concrétude que l'architecture se donne pour tâche d'établir l'homme sur la terre et de lui ouvrir un monde, pour reprendre ici la terminologie de Heidegger dont Ingarden est proche à la fin du chapitre 3 de L'œuvre architecturale. C'est aussi, à son estime, cette concrétude en laquelle s'incarne la destinée humaine qui lui confère une valeur plus haute que celle des autres activités artistiques.

Des observations similaires s'imposent à propos du spectateur de l'architecture. Ingarden n'évoque jamais l'emploi que ce dernier peut faire de son imagination devant ou dans un bâtiment. C'est que le regard qu'il porte sur l'œuvre édifiée est, lui aussi, conditionné, en ultime ressort, «par l'ordre des masses ${ }^{6}$ que nous venons à l'instant d'évoquer. Que, par la variété des perspectives qu'il peut prendre sur lui, l'observateur ait la capacité de faire

\footnotetext{
${ }^{1}$ R. Ingarden, op. cit., p. 96.

${ }^{2}$ Ibid., p. 96-97.

${ }^{3}$ Ibid., p. 96.

${ }^{4}$ Ibid., p. 97.

${ }^{5}$ Ibid., p. 106.

${ }^{6}$ Ibid., p. 56.
} 
apparaître un bâtiment donné sous de multiples aspects, ainsi qu'Ingarden le fait valoir au chapitre 6 de son ouvrage - aspects qui, précisons-le, sont « de nature proprement perceptive $»^{1}-$, n'enlève rien au fait que, d'après lui, les seules perceptions valables soient celles qui conduisent à des «actes de reconstruction $»^{2}$ d'un complexe architectural aux propriétés physiques et fonctionnelles déterminées que de tels actes ont justement pour finalité de révéler de la manière la plus conforme qui soit. C'est que la relation esthétique que nous entretenons avec un bâtiment "n'est pas une simple fiction ni une formation arbitraire quelconque de l'observateur percevant $»^{3}$, comme Ingarden le stipule avec vigueur. Il importe donc qu'elle s'appuie sur la prise en compte du bâtiment réel car c'est en ce dernier que se situe «le fondement de son être et de sa détermination. ${ }^{4}$

\section{Le modernisme ou l'épokhè architecturale}

Il serait absurde de nier l'importance qu'il convient de reconnaitre, avec Ingarden et tant d'autres avant (que l'on songe à Vitruve) et après lui (Henri van Lier que nous citions plus haut), aux contraintes physico-techniques et aux dispositions pratiques qui s'imposent d'entrée de jeu à la composition architecturale. Par contre, il ne va pas de soi que la prise en considération des propriétés physiques de l'objet architectural et la mise en avant de la destination fonctionnelle qu'il doit remplir conduisent nécessairement à mésestimer ou à minimiser le rôle que l'imagination peut être amenée à tenir dans l'instauration et l'appréhension des œuvres construites. Tout dépend de la manière dont la matérialité et la fonctionnalité de l'édifice sont pensées et aussi du statut qui leur est accordé.

De ce point de vue, la rupture décisive introduite au début du $20^{\mathrm{e}}$ siècle dans l'histoire de l'architecture par le modernisme est riche d'enseignements. Le choc produit par une telle rupture n'a pas échappé à Ingarden, reconnaissons-le. Il a su détecter avec acuité dans le mouvement moderne une prédilection pour « la prédominance du spatial dans la construction de l'œuvre » ainsi que pour la « simplification de la construction d'ensemble $»^{5}$. Il a vu que le résultat de cette simplification aboutissait à la disparition « des

\footnotetext{
${ }^{1}$ Ibid.

${ }^{2}$ Ibid., p. 50.

${ }^{3}$ Ibid.

${ }^{4}$ Ibid.

${ }^{5}$ Ibid., p. 94.
} 
moments d'ornementation qui ne sont pas architecturaux en leur essence ${ }^{1}$. Et qu'une telle disparition - Adolf Loos en avait fait son credo - devait contribuer à renforcer la lisibilité de la structure spatiale de l'œuvre construite. Il a su apprécier, enfin, l'usage fait par les architectes modernes des formes géométriques dont la sobriété laisse apparaître clairement « les lois de la statique des masses lourdes », tout en s'associant de façon harmonieuse « avec le but fonctionnel du bâtiment $»^{2}$. Autant d'éléments qui ont retenu son attention et qui l'ont poussé à voir dans l'irruption du modernisme le moment d'accomplissement de l'essence de l'art de bâtir ${ }^{3}$. Patricia LimidoHeulot a examiné tout cela avec beaucoup de précision dans son introduction à $L$ 'œeuvre architecturale ${ }^{4}$. Nous y renvoyons donc le lecteur.

Mais, il nous semble qu'il y a, dans le modernisme architectural, quelque chose de plus radical que l'adéquation des volumes élémentaires de la géométrie à la finalité utilitaire de l'architecture et à «la statique des masses lourdes », quelque chose de plus exigeant, de plus intransigeant que la conciliation de la forme avec la fonction et sa traduction visible, pour parler comme Schopenhauer, du combat provoqué par les forces de la pesanteur avec celles qu'offre la résistance ${ }^{5}$. À notre avis, ce qui fait l'audace du modernisme, ce qui le rend éminemment singulier, c'est d'avoir mis en œuvre ce qu'il n'est pas inapproprié d'appeler une épokhè architecturale.

Qu'on ne s'y trompe pas, cette réduction architecturale n'est pas issue en droite ligne d'une lecture de Husserl ou d'une influence directe de la phénoménologie sur la façon dont l'architecture s'est pensée à l'aube du $20^{\mathrm{e}}$ siècle. Elle est parallèle et quasiment contemporaine à la réduction phénoménologique telle que Husserl en a engagé le procès sur le plan philosophique, dès 1907, avec L'idée de la phénoménologie. De même qu'elle est proche parente et presque concomitante de l'épokhè que Cézanne, Braque et Picasso ont mis en œuvre dans le champ pictural durant la seconde moitié du $19^{\mathrm{e}}$ siècle et au début du $20^{\mathrm{e}}$ siècle. Épokhè picturale sur laquelle MerleauPonty ${ }^{6}$ et Éliane Escoubas ${ }^{7}$ ont jeté un regard éclairant. La proximité des

\footnotetext{
${ }^{1}$ Ibid.

${ }^{2}$ Ibid.

${ }^{3}$ Ibid.

${ }^{4}$ Cf. P. Limido-Heulot, Introduction à L'œuvre architecturale, op. cit., p. 28 et sq.

${ }^{5} \mathrm{Cf}$. A. Schopenhauer, Le monde comme volonté et comme représentation, 1818, Paris, PUF, 2009, p. 275.

${ }^{6}$ Cf. « Le doute de Cézanne », dans Sens et non-sens, Paris, Nagel, 1948, p. 15-49; cf. aussi L'OEil et l'Esprit, Paris, Gallimard, 1964.

${ }^{7}$ Cf. «L'épokhè picturale : Braque et Picasso », dans La Part de l'œil, nº 7, 1991, p. $189-203$.
} 
deux épokhè, picturale et architecturale, notons-le en passant, étant d'autant plus notable que l'architecture moderne a reçu du cubisme, aux dires de Le Corbusier, ses «ferments fécondants $»^{1}$.

Avant de nous y attarder plus en détail, ce que, par anticipation, nous désignons par épokhè architecturale, c'est la rigueur avec laquelle l'architecture moderne s'est interrogée théoriquement, mais, surtout, pratiquement, sur le sens de ce qu'est, en son fonds ultime, l'acte d'édification, sur ce à quoi cet acte se « réduit » lorsqu'on l'envisage dans sa radicalité la plus pure. Or, il apparaît que la fonction et la lourdeur des masses que la forme architecturale ne doit pas occulter, mais, à l'inverse, permettre de saisir dans « une appréhension correcte $»^{2}$, n'en sont pas, au contraire de ce que pense Ingarden, le dernier ressort, le principe cardinal. Ce que, dans ses expérimentations les plus audacieuses, les plus risquées même, le modernisme a cherché à imposer - et que pressent Ingarden en parlant de la " prédominance du spatial dans la construction », mais sans prendre, à ce qu'il nous semble, la pleine mesure de ce phénomène - , ce n'est pas seulement la prévalence de l'espace sur les parties solides de la construction et leur fonction, mais, plus encore, sa libération totale, en tout cas maximale, sa fluidification la plus extrême. Ce vers quoi le modernisme a idéalement tendu, ce qu'il s'est efforcé de promouvoir par sa stratégie de la «table rase », c'est un espace vide, isotrope, qui n'a plus ni centre ni pourtour, qui n'est plus qu'une masse d'air traversant sans obstacle la construction, ainsi que les travaux de nombreux spécialistes l'ont mis en évidence ${ }^{3}$.

En vue de donner toute sa place à la vacuité continue de l'espace, de ne pas limiter son amplitude, les représentants du mouvement moderne ont emprunté deux voies réductrices : 1) la "réduction» de la composante matérielle de la construction architecturale, sa dématérialisation, l'abolition de sa masse pesante, bref, la dissolution de sa substantialité "réduite», précisément, à une simple ossature porteuse légère, peu encombrante et indépendante de toute césure interne de l'aire d'habitation autant que de son enveloppe extérieure, laquelle, par voie de conséquence, devient libre de recevoir n'importe quelle apparence ; 2) la « réduction » de la fonctionnalité du bâtiment mise en suspens, laissée indéterminée, indéfinie, autrement dit,

\footnotetext{
${ }^{1}$ Le Corbusier, «Entretien avec les étudiants des écoles d'Architecture », 1942, dans La Charte d'Athènes, 1941, Paris, Minuit, 1957, p. 172.

${ }^{2}$ R. Ingarden, op. cit., p. 95 , note 1.

${ }^{3}$ Cf. S. Giedion, Espace, temps, architecture, $5^{\mathrm{e}}$ éd., 1966, Paris, Denoël, 2004, ch. 6, p. 255-397 ; C. Norberg-Schultz, La signification dans l'architecture occidentale, 1974, Wavre, Mardaga, 1977, ch. 11, p. 357-388.
} 
offerte à la disposition de l'utilisateur libre d'articuler, selon ses propres besoins, le vide spatial autour duquel s'organise la construction moderne.

L'œuvre architecturale moderne est donc, pour le dire encore avec d'autres mots, une « œuvre ouverte», au sens que Umberto Eco prête à cette expression. Le sémioticien italien désigne par-là, on le sait, toutes les productions artistiques réclamant, pour être ce qu'elles sont, l'intervention d'un auteur, bien sûr, mais aussi l'intervention de ceux auxquels elles s'adressent sans que leur identité n'en soit pour autant menacée, mais, tout au contraire, incessamment enrichie et réinventée ${ }^{1}$. Cette double intervention créative mobilise les ressources de l'imagination telle que la phénoménologie de Husserl la définit et que Eco fait explicitement entrer en résonnance avec sa poétique de l'œuvre ouverte. Dans le premier chapitre de L'œuvre ou$v_{\text {verte }}{ }^{2}$, il compare en effet l'œuvre d'art ouverte avec la perception phénoménologique des objets du monde comme synthèse inachevée, inaboutie, et où l'imagination, à tout moment, peut venir prendre en charge l'accomplissement des esquisses en attente d'être délivrées de leur invisibilité, des apparitions potentiellement actualisables, ainsi qu'on le rappelait en commençant.

\section{Ingarden et la structure stratifiée de l'œuvre d'art}

Avant de nous pencher plus précisément sur ce qu'il convient d'entendre par le déploiement moderne d'une épokhè architecturale, revenons, comme nous l'annoncions au début de ce développement, à l'examen auquel Ingarden procède de la structure stratifiée de l'œuvre d'art. C'est à Husserl qu'il doit la découverte de cette structure. La conscience d'image, qui fait l'objet de nombreuses études contenues dans les textes composant le volume XXIII des Husserliana, en est la parfaite illustration. La conscience d'image est la conscience qui s'attache à l'image dotée d'une réalité physique. Cette image est composée de trois couches ou de trois strates qui se superposent les unes sur les autres ${ }^{3}$. La réalité physique de l'image est la « chose-image » qui est perçue en tant qu'objet réel (le tableau, la gravure, etc.). La chose-image n'est pas, à proprement parler, l'image, mais son support, le lieu où elle s'incarne, incarnation qu'il importe de ne pas confondre avec son individualisation. Ce que nous appréhendons dans l'acte accompli par la

${ }^{1}$ Cf. U. Eco, L'œuvre ouverte, 1962, Paris, Seuil, 1965.

${ }^{2}$ Ibid., p. 31-32.

${ }^{3}$ Nous renvoyons à « Phantasia et conscience d'image », op. cit., § 9, p. 63-65. 
conscience imageante, en prenant appui sur la perception de la chose-image, c'est «l'objet-image». Celui-ci est considéré par Husserl comme un « irréel », un « néant ». Il se manifeste à travers la manière dont les matières (formes et couleurs si l'on a affaire à un tableau, par exemple) qui composent la chose-image ont été organisées, agencées par celui qui en est le producteur. Cette organisation nous donne à son tour accès au "sujet-image", c'est-à-dire à ce que figure l'objet-image. À l'instar de ce dernier, le sujetimage est également un irréel. Objet-image et sujet-image, représentant et représenté iconiques, constituent à eux deux le noème de l'image physique. Ils sont les objets visés intentionnellement par l'imagination sur la base d'un existant dont l'acte percevant atteste la présence réelle.

Le modèle de cette structure stratifiée de l'image possédant un soubassement matériel et, de façon plus générale, de l'œuvre d'art, réside, chez Husserl, dans l'intentionnalité langagière, dans la littérature au sens large du terme. Il en aborde le fonctionnement dès les Recherches logiques. Dans cet ouvrage, il est amené à distinguer, en droit, le substrat (sonore ou graphique) des signes linguistiques de leur teneur idéale en signification, une signification qui n'appartient jamais tout-à-fait au signe qui lui sert de véhicule en vertu précisément de son idéalité, laquelle lui donne le pouvoir d'être réitérée ailleurs, dans le corps d'un autre signe auquel elle n'appartiendra pas plus qu'au précédent.

Lorsque Ingarden aborde le problème de l'architecture, la question inaugurale qu'il se pose est celle de savoir si pareille structure est en mesure de s'appliquer à des objets qui frappent immédiatement, ainsi qu'on l'a vu plus haut, par leur épaisseur ontologique: pouvons-nous, se demande-t-il d'emblée dans L'œuvre architecturale, tenir un édifice «pour quelque chose qui n'est pas réel ? ${ }^{1}$. La réponse qu'il apporte à cette interrogation se décompose en deux temps qu'il convient de parcourir.

Dans un premier temps, Ingarden invite à la circonspection, en l'occurrence à ne pas se laisser induire en erreur par la présence massive de l'architecture sur laquelle on a suffisamment insisté jusqu'ici. Les propriétés physiques d'un édifice ne sont pas ses seules propriétés, indique-t-il. En tant qu'objet réel, il est à la base d'une série de prises d'attitudes subjectives, d'actes de conscience divers le donnant à saisir sous des aspects différents, lesquels révèlent d'autres qualités que les qualités matérielles imputables à sa réalité d'objet effectif. Ainsi, le fidèle entrant dans une église prendra soin de marcher à pas feutrés, de respecter le silence régnant dans le sanctuaire qui l'accueille. C'est qu'il arpente un espace symbolique avant d'arpenter la

${ }^{1}$ R. Ingarden, op. cit., p. 40. 
réalité spatiale du bâtiment qui lui sert d'abri. Par contre, le maçon chargé de l'entretien ou de la restauration de cet espace ne se privera pas d'y faire du bruit, parce que, signale Ingarden, «il ne mène pas son travail sur l'église mais sur le bâtiment réel. ${ }^{1}$ Dans l'attitude esthétique, c'est-à-dire dans la contemplation, on assiste à un effacement similaire de la chose édifiée. Celleci, observe Ingarden, «se retire en quelque sorte à l'arrière-plan $»^{2}$ pour laisser place à une variété d'appréhensions perceptives - « en principe une infinité $»^{3}-$, faisant fluctuer sans arrêt l'apparition de l'entité architecturale, laquelle n'est jamais donnée de façon globale et immédiate.

Ingarden reconnaît donc l'existence de deux couches stratifiées composant l'œuvre d'architecture : une couche objective faite de la chose réellement existante en quoi consiste le bâtiment dans sa réalité matérielle et une couche subjective, qui s'ajoute à la première et qui est constituée de l'ensemble des "concrétisations », c'est-à-dire des appréhensions diverses dont l'édifice est la cible de notre part. Il y a, donc, d'un côté, l'objet réel, unique, garantissant le fondement ontique de l'œuvre architecturale, son incarnation, et, de l'autre, l'objet intentionnel, multiple, en ce qu'il résulte de la pluralité de nos perceptions en tant qu'observateurs et utilisateurs de l'objet architecturalement incarné.

De ces deux couches sur lesquelles repose l'ontologie de l'œuvre architecturale, Ingarden commence - quoique de manière réservée - par accorder la prévalence à la seconde : " l'attitude subjective et les actes de conscience correspondants constituent le second fondement ontologique (de l'objet architectural, le premier étant sa factualité), et peut-être le plus important $»^{4}$. Ce passage est le seul où se manifeste la primauté de la deuxième strate ontologique, la strate subjective, sur laquelle se fonde, en partie, l'objet architecturé. Ailleurs, et c'est le second temps de la réponse d'Ingarden, il accorde sa préférence à la première strate, la strate objective, sans, bien sûr, que la deuxième strate ne soit évincée. Mais il est notable que cette dernière soit alors systématiquement subordonnée à la détermination factuelle et fonctionnelle de l'architecture. Nous nous contenterons de citer deux extraits ${ }^{5}$ de L'œuvre architecturale où se signale avec netteté la priorité de la couche objective sur la couche subjective et la relation de dépendance de celle-ci vis-à-vis de celle-là :

\footnotetext{
${ }^{1}$ Ibid., p. 45.

${ }^{2}$ Ibid., p. 41.

${ }^{3}$ Ibid., p. 55. Soyons attentifs à la nuance du « en principe ».

${ }^{4}$ Ibid., p. 46. Nous soulignons.

${ }^{5}$ Il en existe d'autres : cf. p. 60, p. 61 et p. 118.
} 
Ce qui est important dans l'œuvre architecturale, c'est la couche objectale, la forme spatiale du corps de l'œuvre (...). Les aspects servent surtout à l'expression de cette forme et de ses différents détails, et ils ne doivent pas se rendre indépendants de la totalité de l'œuvre.

Dans l'œuvre architecturale, c'est la couche objectale (gegenständliche Schicht), c'est-à-dire la masse tridimensionnelle et sa forme, qui constitue le facteur structurel et phénoménal le plus important et sur le seul fondement duquel se constituent les qualités spécifiques douées de valeur esthétique ${ }^{1}$.

La soumission de la couche aspectuelle à la couche objectale entraîne, de la part d'Ingarden, sur le plan artistique, la condamnation de tout style architectural visant à la dissimulation de l'organisation intrinsèque des volumes et de leurs propriétés physiques. Ainsi en va-t-il du baroque et de son usage du trompe-l'œil, du rococo ou de l'art nouveau dont les décors foisonnants « ont pour effet de rendre presque impossible l'émergence des masses principales et en conséquence de présenter un élément déconcertant dans la structure de la totalité $»^{2}$. Tout décalage entre l'apparition phénoménale et la constitution ontologique du bâtiment est donc fermement condamné par Ingarden.

Le modernisme est, quant à lui, parvenu à éviter cet écueil. Lui seul, avance Ingarden, nous l'évoquions il y a quelques instants, «a compris l'essence de l'œuvre architecturale dans sa logique interne $»^{3}$, parce que lui seul est parvenu à concilier, dans une parfaite transparence, l'expression de la forme avec le traitement rationnel des nécessités pratiques et la disposition des masses volumétriques.

Que son admiration pour le modernisme l'ait par ailleurs incité à mettre l'accent, dans ses analyses, sur la dynamique de l'espace architectural est incontestable. Il suffit de se reporter au chapitre ultime de son ouvrage pour s'en apercevoir. Il y parle de « circulation » et de «promenade à l'intérieur de l'œuvre $»^{4}$, en des termes qui ne sont pas sans faire écho aux propos de Le Corbusier ${ }^{5}$. Influencé, plus que certainement, par celui-ci, il y soutient l'idée que l'art architectural est un art temporel et qu'il est de la plus haute importance que l'entité bâtie puisse être, on l'a vu, « saisie et contemplée de

\footnotetext{
${ }^{1}$ Ibid., p. 58.

${ }^{2}$ Ibid., p. 93.

${ }^{3}$ Ibid., p. 94.

${ }^{4}$ Ibid., p. 113.

Cf. Le Corbusier, Euvres complètes 1910-1929, Zurich, Les Éditions d'Architecture, 1964, p. 60 ; CEuvres complètes 1929-1934, Zurich, Les Éditions d'Architecture, 1964, p. 24.
} 
différents côtés ou points de vue successifs ${ }^{1}{ }^{1}$. Approche cinématique de l'œuvre bâtie que Le Corbusier n'a cessé de défendre en comparant l'architecture à une "symphonie » dont l'unité ne peut être saisie «qu'au fur et à mesure que les pas nous portent, nous placent et nous déplacent $»^{2}$.

Mais, tout en avalisant les avancées du mouvement moderne en ce qui concerne la dimension mouvante de l'espace construit, il ne peut cependant s'empêcher de tempérer la portée de cette dernière. Qu'un bâtiment puisse être parcouru et modifié dans ses apparences au gré de nos déambulations, que sa configuration puisse ainsi prendre plusieurs profils, il reste néanmoins fondamentalement un objet inerte: l'architecture " est en soi-même une formation immobile $»^{3}$. Il ne saurait donc être question, pour Ingarden, au contraire de Henri van Lier, convoqué antérieurement, que le spectateur soit le «centre mobile » ${ }^{4}$ de l'espace architectural. Si celui-ci possède bien un centre, ce centre doit être fixe : le cours changeant de nos saisies visuelles, annonce Ingarden, est « un jeu qui est ultimement relatif à un unique système (...) de formes corporelles ${ }^{5}$. Ce qui l'amène à penser que, s'il y a bien une «vie », une « rythmique ${ }^{6}$ de l'œuvre construite, il faut tout de même que cette vie ou que cette rythmique renvoient, en dernière instance, au « visage originel $»^{7}$ de cette œuvre, qu'elles donnent lieu à « une concrétisation la plus adéquate possible à l'œuvre $»^{8}$. Tels sont les derniers mots du dernier chapitre de L'ouvre architecturale où triomphe, on le constate une fois de plus, le réalisme sur l'idéalisme, l'objet réel sur l'objet intentionnel, l'ontologie sur la phénoménologie de l'œuvre d'art.

Or, il apparaît que le modernisme, dans ses innovations les plus radicales, les plus téméraires, s'est plu à introduire dans l'œuvre architecturale la mobilité qu'Ingarden prêtait seulement à son spectateur et à ses vécus perceptifs. Cette mobilité inhérente à la construction même, formant le cœur de l'épokhè architecturale à laquelle nous arrivons maintenant, surenchérit sur celle de ses utilisateurs et de ses observateurs tout en sollicitant, de manière intense, leur capacité imaginative, leur aptitude à articuler, à ordonner un espace habitable qui est devenu, avec le mouvement moderne, comme

${ }^{1}$ R. Ingarden, op. cit., p. 113.

${ }^{2}$ Le Corbusier, "Entretien avec les étudiants des écoles d'Architecture», op. cit, p. 156

${ }^{3}$ R. Ingarden, op. cit., p. 112.

${ }^{4}$ H. van Lier, op. cit., p. 232.

${ }^{5}$ R. Ingarden, op. cit., p. 114.

${ }^{6}$ Ibid.

${ }^{7}$ Ibid., p. 118.

${ }^{8}$ Ibid., p. 117. 
il en a été question ci-dessus, un espace vacant et homogène, nu et indéfini, par conséquent un espace modulable, transformable à l'infini, un espace potentiellement en perpétuelle mutation. Dans le domaine architectural moderne, dont le leitmotiv est la planification libre, le possible prime sur le réel, la virtualité spatiale sur son actualité, sur l'effectivité de son organisation. Husserl, de son côté, n'affirmait-il pas que la constitution des objets livrés à la perception "se réfère d'abord à une conscience individuelle possible par essence, puis également à une conscience communautaire possible $»^{1}$ ? Et cela, on l'a déjà dit, parce que la chose perçue demeure, en permanence, «ultérieurement déterminable » ${ }^{2}$, toujours en état d'être donnée dans son intégralité sans jamais l'être, c'est-à-dire toujours en réserve d'ellemême et, partant, offerte aux prises des actes de l'imagination qui vient « suppléer» son incomplétude.

\section{Le Corbusier et Mies van der Rohe : la maison « Dom-ino» (1914) et la Maison Farnsworth (1946-1951)}

Les œuvres de Le Corbusier et de Mies van der Rohe nous serviront d'exemples magistraux de la mise en application de l'épokhè dans le champ architectural. Chez ces deux figures éminentes du modernisme, contrairement à ce qui a pu être dit, le mot d'ordre n'est pas tant : "La forme suit la fonction » de Sullivan que «less is more », "moins c'est plus ». Il s'agit là d'un aphorisme attribué à Mies van der Rohe, mais dont il n'est à vrai dire pas l'auteur. En revanche, dans "Immeuble de bureau », un texte paru en 1923 dans la revue d'avant-garde $G$ (lettre initiale de Gestaltung), il fait de l'adage suivant l'emblème de son architecture : «Le maximum d'effet avec le minimum de moyens ${ }^{3}$. Si cet adage résume la ligne conductrice de son activité édificatrice, il résume tout aussi bien celle suivie par Le Corbusier dès le début de sa carrière. Pas de traces de la formule "less is more » chez ce dernier non plus. Mais, par contre, une déclaration qui, à défaut d'en

\footnotetext{
${ }^{1}$ E. Husserl, Idées directrices pour une phénoménologie, 1913, Paris, Gallimard, $1950, \S 135$, p. 453. Nous soulignons.

${ }^{2}$ Ibid.

${ }^{3}$ M. van der Rohe, «Immeuble de bureau », 1923, dans Fritz Neumeyer, Mies van der Rohe. Réflexions sur l'art de bâtir, Paris, Le Moniteur, 1996, p. 241. Dans "Conversation avec Mies van der Rohe», 1958, un entretien avec Christian Norberg-Schultz, Mies van der Rohe déclare avoir « révisé la formule de Sullivan », ibid., p. 339 .
} 
traduire la lettre, en traduit l'esprit: «la loi d'Économie gère impérativement nos actes et nos pensées $»^{1}$.

La maison "Dom-ino » (« domus » = maison ; ino = innovation) est, chez Le Corbusier, l'expression précoce de cette «loi d'Économie», de simplification. Elle aboutit à une vision spatialement épurée de l'habitation. Le concept de ce procédé constructif est posé en 1914 dans des circonstances particulières $^{2}$. Il s'accorde au nouvel état d'esprit que Le Corbusier entend contribuer à répandre par ses écrits comme par ses réalisations. Celui-ci consiste, notamment, à se débarrasser des «concepts immobiles de la maison $»^{3}$. Pour y parvenir, deux conditions doivent être réunies : «La plus active imagination » et «la plus sévère discipline ${ }^{4}$. «La plus sévère discipline », tout d'abord, car il s'agit de concevoir, à nouveaux frais, une structure habitable rigoureuse, simple et sobre. En ce qui concerne la maison « Dom-ino », cette structure consiste en une ossature portante autonome, sans relation, donc, avec le programme fonctionnel de la maison et la forme externe qu'elle revêtira. Elle rassemble trois dalles en béton superposées faisant office de planchers et de plafonds. Ces trois dalles sont reliées entre elles par des escaliers et soutenues par six poteaux très minces (anticipation des pilotis constituant l'un des cinq points de l'architecture nouvelle thématisés par Le Corbusier en 1927). Fabriqués industriellement selon des proportions standardisées, ces éléments modulaires sont combinables entre eux, à l'image des pièces du jeu de dominos dont ils évoquent le mode d'assemblage : leur association et leur extension, indéfinies, sont aussi bien verticales qu'horizontales. De sorte que la maison « Dom-ino » est un prototype qui ne possède pas de limites a priori déterminées. Quant à l'espace dégagé par la surface habitable, il est vide, pur néant à peine obturé par la présence de piliers s'apparentant au squelette de l'édifice. Applicable à tout genre de construction, Le Corbusier résume son invention comme suit : " On a (...) conçu un système de structure - ossature - complètement indépendant des fonctions du plan de la maison $»^{5}$. Ce système autorise «d'innombrables

${ }^{1}$ Le Corbusier, Vers une architecture, 1923, Paris, Champs-Flammarion, 1995, p. XX.

${ }^{2}$ Les destructions de la Première Guerre mondiale dans les Flandres, en septembre 1914.

${ }^{3}$ Ibid., p. 193.

${ }^{4}$ Ibid., p. 36.

${ }^{5}$ Le Corbusier, Euvres complètes 1910-1929, op. cit., p. 23. 
combinaisons de dispositions intérieures $»^{1}$, tout en faisant naître « un sentiment neuf de l'esthétique architecturale $»^{2}$.

Cette économie constructive, on le comprend sans peine, fait appel ensuite à « la plus active imagination », puisque mission est dévolue à l'habitant de ce lieu vierge de toute structuration préalable, spatialement flexible et extensible, d'inventer lui-même l'organisation de son élément vital. L'homogénéité et la continuité de l'espace, aussi bien interne (il n'y a pas de murs séparateurs) qu'externe (il n'y a pas de surfaces de revêtement), que rien ne vient entraver, réclame de sa part qu'il vienne différencier une étendue qui, dans l'attente de sa partition, est inhabitable ou, plus exactement, est la matrice de toute habitabilité concevable. L'usager générera un mode d'habitation, son mode d'habitation, par des gestes constructifs simples, élémentaires, gestes à quoi se "réduit», selon Le Corbusier, l'acte architectural primitif $^{3}$ : en obturant, au moyen de parois, le dénuement de l'espace, et en y perçant des ouvertures. Le tout sans contrainte, sans imposition préalable, sans prescription programmatique. C'est dire qu'il lui faut imaginer ce que cette spatialité ouverte lui offre de possibilités fonctionnelles à venir et à faire, ensuite, advenir réellement. Spatialité qu'il pourra en plus modifier dans l'avenir comme bon lui semble, puisque Le Corbusier préconise d'employer, en guise d'éléments séparateurs de l'espace intérieur, des cloisons démontables, amovibles, favorisant le jeu d'une réélaboration incessante de la topographie architecturale, sans cesse ajustable, adaptable aux besoins changeants de son occupant: "Les cloisons légères, note-t-il, peuvent être déplacées dans la suite et le plan peut être transformé facilement $»^{4}$. La séparation spatiale n'est donc jamais, chez Le Corbusier, on en prend acte, une clôture définitive.

Chez Mies van der Rohe, ni le plan ni la fonction ne sont, pas plus que chez Le Corbusier, prescrits d'avance. " Afin de permettre à chacun de réaliser ses idées dans la plus grande liberté, j'ai renoncé aux directives et aux contraintes d'un programme », consigne-t-il dans la Préface de Architec-

\footnotetext{
${ }^{1}$ Ibid.

${ }^{2}$ Ibid., p. 26. Renouant avec l'idéal antique du Kalos kagathos, Le Corbusier note à propos de l'esthétique nouvelle qu'il appelle de ses vœux et que promeut la maison «Dom-ino » : «C'est ici que le principe de la maison en série montre sa valeur morale : un certain lien commun entre l'habitation du riche et celle du pauvre » (Vers une architecture, op. cit., p. 195).

${ }^{3} \mathrm{Cf}$. Vers une architecture, op. cit., ch. III, p. 53-64.

${ }^{4}$ Le Corbusier, Euvres complètes 1910-1929, op. cit., p. 28.
} 
ture et logement (1927) ${ }^{1}$. Et de reconnaître, dans la foulée, que l'heure est venue de faire droit au «bâtiment à ossature », parce que, mieux qu'aucun autre, il « permet une conception rationnelle et laisse entièrement libre l'organisation intérieure de l'espace $»^{2}$. Ajoutant, toujours dans le même texte, que les parois coulissantes contribuent grandement à cette plasticité spatiale $^{3}$ qui est le problème dominant pour Mies van der Rohe et non, on s'en rend parfaitement compte au vu de ses déclarations, celui de la fonctionnalité, laquelle n'est pas négligée, bien entendu, mais assouplie, "démocratisée », pourrait-on dire, puisque rendue aux usagers à qui reviennent son invention et sa mise en exercice ${ }^{4}$.

Toutefois, dans sa quête d'un dispositif constructif garantissant l'unité pure, ininterrompue de l'espace habitable, Mies van der Rohe va plus loin que Le Corbusier. Poussant la notion d'habitation jusqu'à son extrême limite, frôlant le seuil en-deçà duquel le bâti s'estompe, voire disparait, il en arrive à altérer la ligne de démarcation séparant l'espace interne de l'espace externe et à fondre le logis dans le milieu de son environnement. Pour ce faire, il privilégie invariablement la peau de verre, la boîte transparente qui laisse apparaître en toute clarté l'ossature porteuse de la construction tout en supprimant pour la vue, à laquelle elle n'offre aucune résistance, le mince écart d'une membrane translucide découpant l'infinité de l'espace en un dedans et un dehors.

Fidèle au principe selon lequel « une construction claire est le fondement du plan libre $»^{5}$, Mies van der Rohe est conduit à rejeter toute indépendance de la forme au regard de la structure ${ }^{6}$, problème que Le Corbusier jugeait, pour sa part, secondaire. Dans l'« Entretien avec les étudiants des écoles d'Architecture », il prétendait qu' " affirmer les éléments de structure, les mettre en évidence (...) n'est (...) qu'une question d'esthétique personnelle sur laquelle il n'est nul besoin de trancher. ${ }^{7} \mathrm{C}^{\prime}$ est ce que démontre Notre-Dame-du-Haut (1950-1955). Chapelle de pèlerinage située près de Ronchamp, dans les Vosges, en France, sa forme sculpturale se

\footnotetext{
${ }^{1}$ M. van der Rohe, Préface de Architecture et logement, 1927, dans Fritz Neumeyer, op. cit., p. 261.

${ }^{2}$ Ibid., p. 263.

${ }^{3}$ Ibid.

${ }^{4}$ Cf. « Que seraient le béton et l'acier sans le vitrage ? », 1933, dans Fritz Neumeyer, op. cit., p. 310.

5 "Conversation avec Mies van der Rohe », op. cit., p. 338.

${ }^{6}$ Cf. « Conférence », date inconnue, dans Fritz Neumeyer, op. cit., p. 320.

${ }^{7}$ Le Corbusier, " Entretien avec les étudiants des écoles d'Architecture », op. cit., p. 164. Notons ici l'antagonisme avec Ingarden.
} 
désolidarise de l'ordonnance interne de l'édifice. Certains critiques, tels que Charles Jencks, par exemple, parlent d'architecture métaphorique et se plaisent à déceler les significations visuelles ambigües suggérées par la coque en porte-à-faux de sa toiture, censée exprimer tantôt deux mains jointes tendues vers le ciel, tantôt les ailes d'un oiseau ou d'un papillon, tantôt encore le couvre-chef de certains hommes d'église ${ }^{1}$. L'imagination symbolique est ici sollicitée. Thématique que nous n'aborderons pas.

Pour en revenir à la boîte de verre qui a les faveurs de Mies van der Rohe, arrêtons-nous sur la Maison Farnsworth (Plano, Illinois, 1946-1951) qui en est, assurément, l'une des manifestations les plus exemplaires. Bâtiment minimaliste, elle représente, pour reprendre les termes de Paolo Amaldi, «le degré zéro de l'architecture en la réduisant à sa plus simple articulation $»^{2}$. Ou, pour citer Fritz Neumeyer, elle est le pendant, dans la sphère du bâti, de " la réduction suprématiste d'un Malevitch » ${ }^{3}$ dont on a le souvenir que, à l'instar de Kandinsky et de Mondrian, il érigeait l'architecture en point suprême d'aboutissement de son entreprise. Autant dire que, tendanciellement en tout cas, cette demeure mène à une épuration de l'architecture, si ce n'est à sa négation, comme maints commentateurs de l'œuvre de Mies van der Rohe l'ont souligné ${ }^{4}$.

De quoi, en sa plus extrême simplicité, en sa stricte minimalité, se compose cette demeure ? De quelques parois vitrées jouant le rôle d'écrans de séparation - mais qui sont tout aussi bien des organes de transition entre le dedans et le dehors ${ }^{5}$. Ces pans de verre sont pris en tenaille par deux dalles horizontales en béton formant la toiture et le plancher de la maison. Ces deux dalles sont elles-mêmes soutenues par huit piliers métalliques

\footnotetext{
${ }^{1}$ Cf. C. Jencks, Le langage de l'architecture post-moderne, 1977, Londres, Academy Editions-Denoël, 1979, p. 48 et sq.

${ }^{2}$ P. Amaldi, Espace et densité. Mies van der Rohe, Gollion, Infolio éditions, 2006, p. 41. Nous soulignons.

${ }^{3}$ F. Neumeyer, op. cit., p. 23. Nous soulignons.

${ }^{4}$ Voir P. Amaldi, op. cit., p. 46.

5 Jean-Claude Cornesse et Michel Prégardien ont, à cet égard, justement mis en relief l'importance des grandes étendues américaines aux horizons sans fin que l'architecte découvre lors de son émigration aux États-Unis, en 1938 (cf. Villas du XX siècle, Liège, Les Éditions de l'Université de Liège, 2010, p. 158). Fritz Neumeyer a, pour sa part, insisté sur l'influence du philosophe Romano Guardini pour qui l'homme et l'univers ne sont que les deux faces d'une seule et même unité supérieure que traduit l'harmonie du paysage et de l'architecture. Mies van der Rohe le connaissait et possédait, dans sa bibliothèque personnelle, les œuvres de ce penseur dont il avait souligné certains passages (cf. Fritz Neumeyer, op. cit., ch. VI, p. 198-236).
} 
situés hors de l'espace d'habitation. Celui-ci est entièrement continu, si ce n'est le cour de la maison occupé par un caisson de bois qui regroupe les deux salles de bain, la penderie, l'âtre et la cuisine. Le reste est vide, offert à la liberté d'imagination et d'organisation de l'occupant du lieu.

Dans la mesure où Mies van der Rohe conçoit son bâtiment « comme un vaste espace unique » garantissant «toutes les flexibilités d'usage $»^{1}$, dans la mesure aussi où, voulant éviter «tout fonctionnalisme étroit», il apporte, dans ses constructions, "l'indispensable espace clos en même temps que la liberté de l'espace ouvert $»^{2}$, c'est à l'occupant qu'il incombe d'agencer son milieu de vie, de l'adapter à ses exigences. Ce faisant, c'est à son style de vie qu'il contribue ${ }^{3}$, comme c'était déjà le cas avec le système « Dom-ino» de Le Corbusier. Pour ces deux architectes, il en va un peu comme si l'objet habité et le sujet habitant se constituaient réciproquement par le geste premier de l'édification qui revient à ouvrir et à fermer l'illimitation de l'espace. Cette ouverture et cette fermeture constituant les deux modalités, dialectiques et intentionnelles, selon lesquelles nous prenons place dans notre environnement en tant que sujets corporels.

On comprend dès lors pourquoi Mies van der Rohe fait du problème spatial, non un problème technique ou utilitaire, mais un problème qui «ne peut être résolu que par les forces créatrices $\gg{ }^{4}$. Celles de l'architecte, cela va de soi. Mais celui-ci se borne à fournir un cadre souple - l'ossature porteuse et le cube de verre - où domine le vide sur le plein et où les fonctionnalités demeurent embryonnaires ${ }^{5}$. Celles qu'est amené à déployer l'usager pour articuler un continuum spatial dont l'ordonnancement doit être imaginé avant et afin d'être réalisé. Car, ici, le corps du bâtiment s'efface au profit de

\footnotetext{
${ }^{1}$ M. van der Rohe, «Un musée pour une petite ville », 1943, dans F. Neumeyer, op. cit., p. 317.

${ }^{2}$ M. van der Rohe, "Maison H., Magdebourg », 1935, dans F. Neumeyer, op. cit., p. 310 .

${ }^{3}$ Le combat pour initier une nouvelle architecture est un « élément de la grande lutte pour de nouvelles formes de vie » (M. van der Rohe, "Conférence », 1927, dans F. Neumeyer, op. cit., p. 264).

${ }^{4}$ M. van der Rohe, «Avant-propos » de Die Form, n 2, 1927, dans F. Neumeyer, op. cit., p. 263.

${ }^{5}$ « J'essaie de faire de mes bâtiments des cadres neutres, dans lesquels les hommes et les œuvres d'art peuvent mener leur vie propre. » («Conversation avec Mies van der Rohe », dans F. Neumeyer, op. cit., p. 340).
} 
l'espace solitaire, ascétique, anonyme ${ }^{1}$. Et l'imagination, dès lors, fait plus que se cantonner à un rôle secondaire ou à n'être qu'une quasi-expérience, elle rend l'espace perceptible en lui conférant une articulation, un rythme, en lui donnant forme et en le rendant, par cela même, habitable.

\section{Dissipation ou schématisation de l'architecture?}

Si l'on admet, avec Merleau-Ponty et Éliane Escoubas, que l'épokhè picturale moderne, entamée d'abord par l'impressionnisme, poursuivie ensuite par Cézanne et les cubistes, consiste dans la disparition de l'objet peint, soit sous ses vibrations colorées (impressionnisme), soit sous la diffraction de ses esquisses simultanées (cubisme), on n'aura pas de difficulté à admettre que l'épokhè architecturale moderne, proche cousine de la précédente, procède à la dilution de l'objet bâti en le dépourvoyant de sa massivité et en ruinant toute destination utilitaire préétablie à laquelle il pourrait répondre. Face à pareil schématisme, Jean-Claude Cornesse et Michel Prégardien se sont demandés si on n'assistait pas purement et simplement à «l'effacement de l'architecture? $»^{2}$. Question qui demeure en suspens. Ce qui est sûr en tout cas, c'est que l'armature - de fer ou de béton - remplit les conditions minimales de possibilité de réalisation de l'architecture. Peut-être même estce en elle que réside son essence et aussi sa beauté dont Mies van der Rohe disait qu'elle était " indéterminable $»^{3}$ ? Au nom de ce radicalisme extrême, en cet abandon assumé de la rigidité et de la fonctionnalité de la construction, c'est à un sujet d'abord imaginant, placé devant la nécessité de participer à l'invention de son habitat et de sa vie d'être spatial, dans une démarche véritablement générative, que les représentants du modernisme, Le Corbusier et Mies van der Rohe en tête, ont, au nom de la liberté, lancé un appel.

\footnotetext{
${ }^{1}$ Mies van der Rohe affirme, par ses constructions, vouloir refléter la «grande tendance à l'anonymat» ("Architecture et volonté de l'époque», 1924, dans F. Neumeyer, op. cit., p. 245) de son époque. ${ }^{2}$ J.-C. Cornesse et M. Prégardien, Villas du XX $X^{e}$ siècle, op. cit., p. 159.

${ }^{3}$ M. van der Rohe, «Bâtir beau et pratique. Pour en finir avec la froideur du fonctionnalisme », 1930, dans F. Neumeyer, op. cit., p. 301. « La question de l'essence de l'architecture est d'une importance cruciale » (M. van der Rohe, « Architecture et volonté de l'époque », op. cit., p. 245).
} 


\section{Bibliographie}

Amaldi P., Espace et densité. Mies van der Rohe, Gollion, Infolio éditions, 2006.

Cornesse J.C. et Prégardien M., Villas $d u X X^{e}$ siècle, Liège, Les Éditions de l'Université de Liège, 2010.

Dufourcq A., La dimension imaginaire du réel dans la philosophie de Husserl, Dordrecht, Springer, 2011.

Eco U., L'œuvre ouverte, 1962, Paris, Seuil, 1965.

Escoubas É., «L'épokhè picturale : Braque et Picasso », dans La Part de l'Eil, n 7 , 1991, p. 189-203.

Giedion S., Espace, temps, architecture, 5 éd., 1966, Paris, Denoël, 2004.

Husserl E., "Phantasia et conscience d'image", 1904/1905, dans Phantasia, conscience d'image, souvenir. De la phénoménologie des présentifications intuitives. Textes posthumes (1898-1925), Grenoble, Millon, 2002.

Husserl E., Idées directrices pour une phénoménologie, 1913, Paris, Gallimard, 1950.

Husserl E., Méditations cartésiennes, 1950, Paris, PUF, 1994.

Ingarden R., L'œeuvre architecturale, 1945, Paris, Vrin, 2013.

Jenks C., Le langage de l'architecture post-moderne, 1977, Londres, Academy Editions-Denoël, 1979.

Le Corbusier, Vers une architecture, 1923, Paris, Champs-Flammarion, 1995.

Le Corbusier, "Entretien avec les étudiants des écoles d'Architecture », dans $L a$ Charte d'Athènes, 1941, Paris, Minuit, 1957.

Le Corbusier, Euvres complètes 1910-1929 et CEuvres complètes 1929-1934, Zurich, Les Éditions d'architecture, 1964.

Limido-Heulot P., Introduction à R. Ingarden, L'œuvre architecturale, 1945, Paris, Vrin, 2013.

Merleau-Ponty M., «Le doute de Cézanne », dans Sens et non-sens, Paris, Nagel, 1948.

Merleau-Ponty M., L'OEil et l'Esprit, Paris, Gallimard, 1964.

Norberg-Schultz C., La signification dans l'architecture occidentale, 1974, Wavre, Mardaga, 1977.

Schopenhauer A., Le monde comme volonté et comme représentation, 1818, Paris, PUF, 2009.

Sullivan L.H., De la tour de bureau artistiquement considérée, 1896, Montréal, B2, 2011.

van der Rohe M., «Immeuble de bureau », 1923, dans Fritz Neumeyer, Mies van der Rohe. Réflexions sur l'art de bâtir, Paris, Le Moniteur, 1996.

van der Rohe M., " Architecture et volonté de l'époque », 1924, ibid.

van der Rohe M., Préface de Architecture et logement, 1927, ibid.

van der Rohe M., « Avant-propos » de Die Form, n 2, 1927, ibid.

van der Rohe M., «Bâtir beau et pratique. Pour en finir avec la froideur du fonctionnalisme », 1930, ibid.

van der Rohe M., «Que seraient le béton et l'acier sans le vitrage ? », 1933, ibid. 
van der Rohe M., « Maison H., Magdebourg », 1935, ibid.

van der Rohe M., «Un musée pour une petite ville », 1943, ibid.

van der Rohe M., "Conférence », date inconnue, ibid.

van der Rohe M., «Conversation avec Mies van der Rohe », 1958, ibid.

van Lier H., Les arts de l'espace, 1959, Tournai, Casterman, 1971.

363

Bull. anal. phén. XIII 2 (2017)

http://popups.ulg.ac.be/1782-2041/ @ 2017 ULg BAP 